\title{
EL PROBLEMA DEL SOBREPESO Y LA OBESIDAD EN EL PERÚ: LA URGENCIA DE UNA POLIITICA DE SALUD PÚBLICA PARA CONTROLARLA
}

\author{
OVERWEIGHT AND OBESITY IN PERU: URGENT NEED TO HAVE A PUBLIC HEALTH \\ POLICY FOR THEIR CONTROL
}

Claudio F. Lanata ${ }^{1,2, a}$

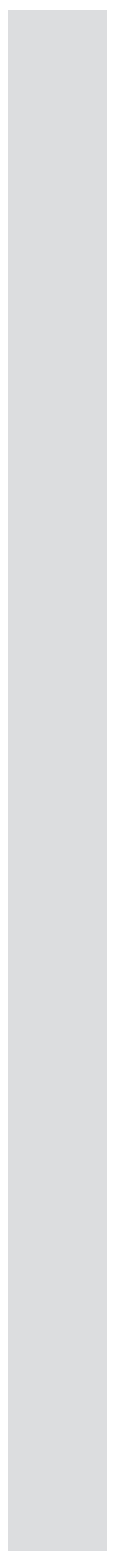

Por muchos años la prioridad nutricional del país ha sido la lucha contra la desnutrición infantil. En un inicio se centró en la lucha contra la desnutrición aguda, manifestada por un bajo peso para la talla del niño o, en sus niveles más extremos, por los cuadros clínicos conocidos como el marasmo o el kwashiorkor. Gracias a los programas nutricionales y al desarrollo socioeconómico del país, la prevalencia de niños con bajo peso para su talla es menor al $1 \%$ y los cuadros clínicos severos casi no se ven. Lo que persiste son niños con baja talla para su edad, mal llamados "desnutridos crónicos" pues representan, mas bien, un retardo en la velocidad de crecimiento producto de una mala calidad de su alimentación en los primeros dos años de vida. Se ha determinado que la baja talla está asociada con una baja ingesta de proteína de origen animal, de calcio (a través de leche o productos lácteos) y de zinc que tenga una alta biodisponibilidad (como a través de carnes o productos marinos).

En forma paralela, también asociada con la mejora económica del país y a los cambios socioculturales ocurridos, el problema del sobrepeso y la obesidad se ha incrementado en forma exponencial, tanto en adultos como en niños, principalmente en zonas urbanas pero también rurales, constituyéndose en un problema de salud pública que no ha sido adecuadamente reconocido y enfrentado. La ayuda alimentaria que el Estado venía proporcionando a la población para evitar la desnutrición, a través de los comedores populares, que consiste generalmente en la distribución de harina blanca, arroz, aceites (no de oliva), fideos o similares, parecería no tener un efecto en la desnutrición y más bien favorecer la presencia de obesidad y sobrepeso. Estos programas deberían rediseñarse para repartir frutas y verduras (lo cual ayudaría a incrementar la producción y el consumo de estos productos), unido a programas de promoción del ejercicio físico. El Programa del Vaso de Leche debería distribuir únicamente leche, idealmente fortificada con hierro, calcio y zinc, para lograr la ingesta recomendada por la Organización Mundial de la Salud (de $160 \mathrm{~kg}$ de leche o productos lácteos per cápita al año, que equivale a medio litro de leche al día), eliminando la distribución de otro tipo de alimentos, como lo recomendara al Ministerio de Salud (MINSA) una comisión multisectorial en el gobierno anterior ${ }^{(1,2)}$. El proyecto de ley "Promoción de la Alimentación Saludable para Niños, Niñas y Adolescentes" que se viene discutiendo en el Congreso para una alimentación saludable ${ }^{(3)}$ debe incorporar las recomendaciones dadas en la sección simposio de este número de la revista y lograr así una mayor efectividad. Entre tanto, el MINSA debería establecer un programa para la prevención y control del sobrepeso y obesidad, y de las enfermedades crónicas no trasmisibles, implementando un sistema regular que evalúe un conjunto de indicadores; estableciéndose metas que sirvan para evaluar la efectividad de programas que se logren implementar. Esta es una agenda urgente, la cual debe ejecutarse sin que se deje de lado el seguir luchando contra las enfermedades infecciosas y otras enfermedades típicas de la transición epidemiológica en que el país se encuentra.

Este número de la Revista Peruana de Medicina Experimental y Salud Pública ha sido dedicado al tema de nutrición, particularmente al sobrepeso y obesidad y su contribución a las enfermedades crónicas no trasmisibles. En el simposio de este número, la nutricionista Reyna Liria ${ }^{(4)}$ nos presenta el problema a nivel nacional, y las tendencias que hacen que el Perú se sitúe como uno de los países en donde dicho problema se ha incrementado rápidamente en la última década, en comparación con otros países de la región. La Dra. Mónica Mispireta ${ }^{(5)}$ hace hincapié en la prevalencia de sobrepeso y obesidad en escolares y cómo este se asocia tanto a un incremento

\footnotetext{
Instituto de Investigación Nutricional. Lima, Perú.

Centro de Investigación de Enfermedades Tropicales, NAMRU 6. Lima, Perú.

Editor invitado, médico infectólogo maestro en Salud Pública

Recibido: 20-07-12 Aprobado: 29-08-12
}

Citar como: Lanata CF. El problema del sobrepeso y la obesidad en el Perú: la urgencia de una política de salud pública para controlarla. Rev Peru Med Exp Salud Publica. 2012;29(3):299-300. 
de la ingesta como a la falta de actividad física, tema que requiere de un abordaje en las escuelas y en las familias. El Dr. Jaime Miranda ${ }^{(6)}$ demuestra cómo la población migrante en el Perú es más vulnerable de sufrir estas enfermedades, incluyendo las enfermedades crónicas no transmisibles, presentando uno de los pocos estudios realizados en el área andina sobre este tema. El Dr. José Saavedra ${ }^{(7)}$ nos hace una excelente revisión de los últimos estudios sobre los determinantes del sobrepeso y obesidad, demostrando claramente cómo este se inicia desde la gestación y, particularmente, en los hábitos alimenticios introducidos por las familias, en los primeros dos años de vida de los niños: la ingesta de alimentos y bebidas azucaradas y la poca ingesta de frutas y verduras son determinantes de los patrones alimenticios que más tarde se asocian con sobrepeso y obesidad.

Finalmente, la nutricionista Hilary Creed y la Dra. Maureen Black ${ }^{\left({ }^{8}\right)}$ nos presentan un resumen de cómo una madre debe alimentar a su niño en los primeros dos años de vida, respondiendo a las señales y avisos que el niño da, para evitar introducir actitudes y patrones de alimentación equivocados, y así no llegar al problema del sobrepeso, la obesidad, o la desnutrición infantil. El manejo adecuado de la alimentación temprana de los niños, particularmente entre los 6 y 24 meses de vida, separando lo que es alimentar de lo que es calmar a un niño para que duerma o deje de llorar; el buscar que el niño participe activamente del acto de comer, y otras claras recomendaciones que se dan en este artículo a las madres, son muy importantes para evitar los problemas nutricionales. Esto, unido a la lactancia materna exclusiva en los primeros seis meses de vida y su continuación hasta el segundo año de vida, permite evitar tanto la desnutrición infantil como el sobrepeso y la obesidad.

La disminución o eliminación de la ingesta de bebidas y alimentos azucarados, así como establecer tempranamente el hábito de la ingesta adecuada de frutas y verduras; la ingesta de lácteos durante toda la niñez y la adolescencia (alcanzando una ingesta promedio mayor a $500 \mathrm{~mL}$ de leche o productos lácteos al día), así como la ingesta diaria de una fuente de proteínas de origen animal (pescados o carnes), deberían constituir los pilares de una alimentación saludable que evite tanto la talla baja y desnutrición infantil como el sobrepeso y la obesidad. El remplazo de la ingesta de harinas blancas con aquellas de alto contenido de fibra, que retarden su absorción (bajando el índice glicémico de la dieta); el incremento de antioxidantes como el aceite de oliva y una ingesta diaria y moderada de vino en la adultez, elementos fundamentales de la llamada dieta mediterránea, junto con frutas, verduras y proteínas de origen animal, permitirían a los peruanos una vida saludable con bajo riesgo de enfermedades crónicas no transmisibles. Se ha tratado de incriminar a la "comida chatarra" como un determinante del sobrepeso y obesidad en el Perú, lo cual no se ha visto en estudios nutricionales que han evaluado la ingesta en varios grupos poblaciones en nuestro medio; por tanto, el obeso en nuestro país come el mismo tipo de alimentos que el no obeso, solo que come más, con raciones más grandes ${ }^{(9)}$. La promoción del ejercicio físico y evitar el sedentarismo en la zona urbana debe ser otro elemento muy importante que debería promocionarse tanto en las escuelas, municipalidades, como por los padres de familia en el hogar.

Conflictos de interés: el autor declara no tener ningún conflicto de interés. Las opiniones expresadas en este artículo son opiniones propias del autor y no representan necesariamente la política o posición oficial del Departamento de Marina, del Departamento de Defensa o el Gobierno de los Estados Unidos de Norteamérica. El autor trabaja parcialmente como contratado por el Ministerio de Defensa de los Estados Unidos y este artículo ha sido preparado como parte de sus funciones oficiales. Debido a esto, el presente no está protegido por leyes de derechos de autor, ya que constituye contribución del gobierno de los Estados Unidos.

\section{REFERENCIAS BIBLIOGRÁFICAS}

1. Resolución Ministerial 139-2007PCM (10 de mayo de 2007).

2. Resolución Ministerial 404-2007PCM (12 de diciembre de 2007).

3. Perú, Presidencia del Consejo de Ministros (PCM). Informe Final del Grupo de Trabajo Multisectorial encargado de proponer mecanismos para coordinar, evaluar y priorizar políticas y medidas sectoriales relacionadas con el programa del vaso de leche. Lima: PCM; 2008.

4. Perú. Congreso de la República. Dictamen en minoría recaído en los proyectos de ley 774/2011-CR, 775/2011-CR, y 1038/2011-CR con un texto sustitutorio mediante el cual se propone la Ley de Promoción de la Alimentación Saludable para Niños, Niñas y Adolescentes. Lima: Congreso de la República; 2012.

5. Liria R. Consecuencias de la obesidad en el niño y el adolescente: un problema que requiere atención. Rev Peru Med Exp Salud Publica. 2012;29(4):357-60.

6. Mispireta ML. Determinantes del sobrepeso y la obesidad en niños en edad escolar en Perú. Rev Peru Med Exp Salud Publica. 2012;29(4):361-5.

7. Miranda JJ, Wells JCK, Smeeth L. Transiciones en contexto: Hallazgos vinculados a la migración rural-urbana en enfermedades no transmisibles en Perú. Rev Peru Med Exp Salud Publica. 2012;29(4):366-72.
8. Saavedra JM, Dattilo AM. Factores alimentarios y dietéticos asociados a la obesidad infantil: recomendaciones para su prevención antes de los dos años de vida. Rev Peru Med Exp Salud Publica. 2012;29(4):379-85.

9. Black MM, Creed-Kanashiro HM. ¿Cómo alimentar a los niños? La práctica de conductas alimentarias saludables desde la infancia. Rev Peru Med Exp Salud Publica. 2012;29(4):373-8.

10. Liria MR, Mispireta ML, Lanata CF, Creed-Kanashiro HM. Perfil Nutricional en escolares de Lima y Callao. Lima: Instituto de Investigacion Nutricional, 2008. 